Canad. Math. Bull. Vol. 62 (2), 2019 pp. 313-326

http://dx.doi.org/10.4153/CMB-2018-017-2

(C) Canadian Mathematical Society 2018

\title{
On the Limiting Weak-type Behaviors for Maximal Operators Associated with Power Weighted Measure
}

\author{
Xianming Hou and Huoxiong $\mathrm{Wu}$
}

Abstract. Let $\beta \geq 0$, let $e_{1}=(1,0, \ldots, 0)$ be a unit vector on $\mathbb{R}^{n}$, and let $d \mu(x)=|x|^{\beta} d x$ be a power weighted measure on $\mathbb{R}^{n}$. For $0 \leq \alpha<n$, let $M_{\mu}^{\alpha}$ be the centered Hardy-Littlewood maximal function and fractional maximal functions associated with measure $\mu$. This paper shows that for $q=n /(n-\alpha), f \in L^{1}\left(\mathbb{R}^{n}, d \mu\right)$,

$$
\begin{gathered}
\lim _{\lambda \rightarrow 0+} \lambda^{q} \mu\left(\left\{x \in \mathbb{R}^{n}: M_{\mu}^{\alpha} f(x)>\lambda\right\}\right)=\frac{\omega_{n-1}}{(n+\beta) \mu\left(B\left(e_{1}, 1\right)\right)}\|f\|_{L^{1}\left(\mathbb{R}^{n}, d \mu\right)}^{q}, \\
\lim _{\lambda \rightarrow 0+} \lambda^{q} \mu\left(\left\{x \in \mathbb{R}^{n}:\left|M_{\mu}^{\alpha} f(x)-\frac{\left.\left.\|f\|_{L^{1}\left(\mathbb{R}^{n}, d \mu\right)} \mid>\lambda\right\}\right)=0,}{\mu(B(x,|x|))^{1-\alpha / n}}\right|>\lambda,\right.\right.
\end{gathered}
$$

which is new and stronger than the previous result even if $\beta=0$. Meanwhile, the corresponding results for the un-centered maximal functions as well as the fractional integral operators with respect to measure $\mu$ are also obtained.

\section{Introduction and Main Results}

Suppose $\beta \geq 0$. Let $\mu$ be a power weighted measure on $\mathbb{R}^{n}$ with $d \mu(x)=|x|^{\beta} d x$. The goal of this paper is to analyze the limiting weak-type behaviors of maximal operators with respect to $\mu$, which is closely related to the best constants of the weak-type endpoint estimates for the maximal operators.

For $0 \leq \alpha<n, f \in L_{\text {loc }}\left(\mathbb{R}^{n}, d \mu\right)$, define the centered maximal operators

$$
M_{\mu}^{\alpha} f(x):=\sup _{r>0} \frac{1}{\mu(B(x, r))^{1-\alpha / n}} \int_{B(x, r)}|f(y)| d \mu(y),
$$

where $B(x, r)$ is a ball centered at $x$ with radius $r$. The uncentered maximal operators are defined by

$$
\widetilde{M}_{\mu}^{\alpha} f(x):=\sup _{B_{x} \ni x} \frac{1}{\mu\left(B_{x}\right)^{1-\alpha / n}} \int_{B}|f(y)| d \mu(y),
$$

where the supremum is taken over all balls $B_{x}$ containing $x$. For $\alpha=0$, we denote $M_{\mu}^{0}$ by $M_{\mu}$ and $\widetilde{M}_{\mu}^{0}$ by $\widetilde{M}_{\mu}$, which is the centered and uncentered Hardy-Littlewood maximal function associated with $\mu$, respectively.

Received by the editors January 5, 2018; revised April 11, 2018.

Published electronically May 24, 2018.

Supported by the NNSF of China (Nos. 11771358, 11471041) and the NSF of Fujian Province of China (No. 2015J01025). Huoxiong Wu is the corresponding author.

AMS subject classification: 42B20, 42B25.

Keywords: limiting weak type behavior, power weight, Hardy-Littlewood maximal operator, fractional maximal operator, fractional integral. 
In particular, for $\beta=0, d \mu(x)=d x$ is the classical Lebesgue measure, and $M_{\mu}^{\alpha}$ (resp., $\widetilde{M}_{\mu}^{\alpha}$ ) is the classical centered (resp., uncentered) Hardy-Littlewood maximal function $M$ (resp., $\widetilde{M}$ ) for $\alpha=0$ and fractional maximal functions $M_{\alpha}$ (resp., $\widetilde{M}_{\alpha}$ ) for $0<\alpha<n$. In 2005, Janakiraman [7] established, among other things, the following limiting weak-type behaviors of $M$ and $\widetilde{M}$ :

$$
\begin{aligned}
& \lim _{\lambda \rightarrow 0+} \lambda m\left(\left\{x \in \mathbb{R}^{n}: M f(x)>\lambda\right\}\right)=\|f\|_{1}, \quad \forall f \in L^{1}\left(\mathbb{R}^{n}\right), \\
& \lim _{\lambda \rightarrow 0+} \lambda m\left(\left\{x \in \mathbb{R}^{n}: \widetilde{M} f(x)>\lambda\right\}\right)=2^{n}\|f\|_{1}, \quad \forall f \in L^{1}\left(\mathbb{R}^{n}\right) .
\end{aligned}
$$

Recently, Ding and Lai [3] extended the above results to the Hardy-Littlewood maximal operator and fractional maximal operators with homogeneous kernels $\Omega$ satisfying the $L_{\alpha}^{q}$-Dini conditions. Moreover, see [2,7] for the corresponding results of singular and fractional integrals with homogeneous kernels. In addition, Huang and $\mathrm{Hu}$ [6], using the basic covering theorem [5, Theorem 1.6] and delicate analysis techniques, proved the following result:

$$
\lim _{\lambda \rightarrow 0+} \lambda v(\{x \in X: M f(x)>\lambda\})=\frac{1}{4}\|f\|_{1}, \quad \forall f \in L^{1}(X, d v),
$$

where $X=(0, \infty)$ with the Euclidean metric $|\cdot|$ and $d v(x)=x d x$, and $M$ is the corresponding Hardy-Littlewood maximal function in $(X,|\cdot|, v)$.

Note that $d \mu(x)=|x|^{\beta} d x$ is doubling, $\left(\mathbb{R}^{n},|\cdot|, \mu\right)$ is a space of homogenous type. We know from $[1,8]$ that $M_{\mu}^{\alpha}$ and $\widetilde{M}_{\mu}^{\alpha}$ are bounded from $L^{1}\left(\mathbb{R}^{n}, d \mu\right)$ to $L^{q, \infty}\left(\mathbb{R}^{n}, d \mu\right)$ for $0 \leq \alpha<n$ and $q=n /(n-\alpha)$. Inspired by the above works, it is natural to explore the limiting weak-type behaviors of $M_{\mu}^{\alpha}$ and $\widetilde{M}_{\mu}^{\alpha}$. To establish the corresponding result, we consider the following more general forms: for $V$ being an absolutely continuous measure on $\mathbb{R}^{n}$ with respect to measure $d \mu$ and $V\left(\mathbb{R}^{n}\right)<\infty$, define

$$
\begin{aligned}
& M_{\mu}^{\alpha} V(x)=\sup _{r>0} \frac{V(B(x, r))}{\mu(B(x, r))^{1-\alpha / n}}, \\
& \widetilde{M}_{\mu}^{\alpha} V(x)=\sup _{B_{x} \ni x} \frac{V\left(B_{x}\right)}{\mu\left(B_{x}\right)^{1-\alpha / n}}
\end{aligned}
$$

for $0 \leq \alpha<n$.

In order to state our results, we will recall and introduce some notation. Let $e_{1}=$ $(1,0, \ldots, 0)$ be a unit vector on $\mathbb{R}^{n}$, let $\mathbb{S}^{n-1}$ be the unit sphere, and let $\omega_{n-1}$ be the surface area of $\mathbb{S}^{n-1}$. Let $d \sigma\left(x^{\prime}\right)$ denote the induced Lebesgue measure of $\mathbb{S}^{n-1}$. In addition, by rotational invariance, we know that $\mu(B(x /|x|, 1))=\mu\left(B\left(e_{1}, 1\right)\right)$, which will be used in the proofs.

Now we can formulate our main results as follows.

Theorem 1.1 Let $\beta \geq 0,0 \leq \alpha<n$, and $q=n /(n-\alpha)$. Suppose that $V$ is an absolutely continuous measure with respect to measure $\mu$ on $\mathbb{R}^{n}$ and $V\left(\mathbb{R}^{n}\right)<\infty$. Then

(i) $\lim _{\lambda \rightarrow 0+} \lambda^{q} \mu\left(\left\{x \in \mathbb{R}^{n}: M_{\mu}^{\alpha} V(x)>\lambda\right\}\right)=\frac{\omega_{n-1} V\left(\mathbb{R}^{n}\right)^{q}}{(n+\beta) \mu\left(B\left(e_{1}, 1\right)\right)}$;

(ii) $\lim _{\lambda \rightarrow 0+} \lambda^{q} \mu\left(\left\{x \in \mathbb{R}^{n}:\left|M_{\mu}^{\alpha} V(x)-\frac{V\left(\mathbb{R}^{n}\right)}{\mu(B(x,|x|))^{1-\alpha / n}}\right|>\lambda\right\}\right)=0$; 
(iii) $\lim _{\lambda \rightarrow 0+} \lambda^{q} \mu\left(\left\{x \in \mathbb{R}^{n}: \widetilde{M}_{\mu}^{\alpha} V(x)>\lambda\right\}\right)=\frac{2^{n+\beta} \omega_{n-1} V\left(\mathbb{R}^{n}\right)^{q}}{(n+\beta) \mu\left(B\left(e_{1}, 1\right)\right)}$;

(iv) $\lim _{\lambda \rightarrow 0+} \lambda^{q} \mu\left(\left\{x \in \mathbb{R}^{n}:\left|\widetilde{M}_{\mu}^{\alpha} V(x)-\frac{2^{(n+\beta) / q} V\left(\mathbb{R}^{n}\right)}{\mu(B(x,|x|))^{1-\alpha / n}}\right|>\lambda\right\}\right)=0$;

(v) $\lim _{\lambda \rightarrow 0+} \lambda^{q} \mu\left(\left\{x \in \mathbb{R}^{n}:\left|\widetilde{M}_{\mu}^{\alpha} V(x)-2^{(n+\beta) / q} M_{\mu}^{\alpha} V(x)\right|>\lambda\right\}\right)=0$.

In particular, for $f \in L^{1}\left(\mathbb{R}^{n}, d \mu\right)$, taking $d V(x)=|f(x)| d \mu(x)$ in Theorem 1.1, we have the following corollary.

Corollary 1.2 Let $\beta \geq 0,0 \leq \alpha<n$, and $q=n /(n-\alpha)$. Then for $f \in L^{1}\left(\mathbb{R}^{n}, d \mu\right)$,

(i) $\lim _{\lambda \rightarrow 0+} \lambda^{q} \mu\left(\left\{x \in \mathbb{R}^{n}: M_{\mu}^{\alpha} f(x)>\lambda\right\}\right)=\frac{\omega_{n-1}}{(n+\beta) \mu\left(B\left(e_{1}, 1\right)\right)}\|f\|_{L^{1}\left(\mathbb{R}^{n}, d \mu\right)}^{q}$;

(ii) $\lim _{\lambda \rightarrow 0+} \lambda^{q} \mu\left(\left\{x \in \mathbb{R}^{n}:\left|M_{\mu}^{\alpha} f(x)-\frac{\|f\|_{L^{1}\left(\mathbb{R}^{n}, d \mu\right)}}{\mu(B(x,|x|))^{1-\alpha / n}}\right|>\lambda\right\}\right)=0$;

(iii) $\lim _{\lambda \rightarrow 0+} \lambda^{q} \mu\left(\left\{x \in \mathbb{R}^{n}: \widetilde{M}_{\mu}^{\alpha} f(x)>\lambda\right\}\right)=\frac{2^{n+\beta} \omega_{n-1}\|f\|_{L^{1}\left(\mathbb{R}^{n}, d \mu\right)}^{q}}{(n+\beta) \mu\left(B\left(e_{1}, 1\right)\right)}$;

(iv) $\lim _{\lambda \rightarrow 0+} \lambda^{q} \mu\left(\left\{x \in \mathbb{R}^{n}:\left|\widetilde{M}_{\mu}^{\alpha} f(x)-\frac{2^{(n+\beta) / q}\|f\|_{L^{1}\left(\mathbb{R}^{n}, d \mu\right)}}{\mu(B(x,|x|))^{1-\alpha / n}}\right|>\lambda\right\}\right)=0$;

(v) $\lim _{\lambda \rightarrow 0+} \lambda^{q} \mu\left(\left\{x \in \mathbb{R}^{n}:\left|\tilde{M}_{\mu}^{\alpha} f(x)-2^{(n+\beta) / q} M_{\mu}^{\alpha} f(x)\right|>\lambda\right\}\right)=0$.

Remark 1.3 Note that for $\beta=0, \mu\left(B\left(e_{1}, 1\right)\right)=\frac{1}{n} \omega_{n-1}$, our conclusions (i) and (iii) in Theorem 1.1 or Corollary 1.2 recover the corresponding results in [7]. Moreover, we remark that conclusion (ii) or (iv) is stronger than conclusion (i) or (iii) in our theorem and corollary (see Remark 2.2). Therefore, even if $\beta=0$, our results (ii) and (iv) are new and interesting.

Also, we consider the generalization of fractional integrals defined by

$$
I_{\mu}^{\alpha} f(x)=\int_{\mathbb{R}^{n}} \frac{f(y) d \mu(y)}{\mu(B(x,|x-y|))^{1-\alpha / n}}, \quad 0<\alpha<n,
$$

(see [4]) and the more general form:

$$
I_{\mu}^{\alpha} V(x)=\int_{\mathbb{R}^{n}} \frac{d V(y)}{\mu(B(x,|x-y|))^{1-\alpha / n}}
$$

where $V$ is an absolutely continuous finite measure on $\mathbb{R}^{n}$ with respect to $\mu$. It follows from [8, Theorem 1] that $I_{\mu}^{\alpha}$ is bounded from $L^{1}\left(\mathbb{R}^{n}, d \mu\right)$ to $L^{n /(n-\alpha), \infty}\left(\mathbb{R}^{n}, d \mu\right)$ as well as from $L^{p}\left(\mathbb{R}^{n}, d \mu\right)$ to $L^{q}\left(\mathbb{R}^{n}, d \mu\right)$ for $1<p<n /(n-\alpha)$ with $1 / q=1 / p-\alpha / n$.

Motivated by the results above, we will establish the following limiting weak-type behaviors of $I_{\mu}^{\alpha}$.

Theorem 1.4 Let $\beta \geq 0,0<\alpha<n$, and $q=n /(n-\alpha)$. Suppose that $V$ is an absolutely continuous measure with respect to measure $\mu$ on $\mathbb{R}^{n}$ and $V\left(\mathbb{R}^{n}\right)<\infty$. Then 
(i) $\lim _{\lambda \rightarrow 0+} \lambda^{q} \mu\left(\left\{x \in \mathbb{R}^{n}: I_{\mu}^{\alpha} V(x)>\lambda\right\}\right)=\frac{\omega_{n-1} V\left(\mathbb{R}^{n}\right)^{q}}{(n+\beta) \mu\left(B\left(e_{1}, 1\right)\right)} ;$

(ii) $\lim _{\lambda \rightarrow 0+} \lambda^{q} \mu\left(\left\{x \in \mathbb{R}^{n}:\left|I_{\mu}^{\alpha} V(x)-\frac{V\left(\mathbb{R}^{n}\right)}{\mu(B(x,|x|))^{1-\alpha / n}}\right|>\lambda\right\}\right)=0$.

By taking $d V(x)=f(x) d \mu(x)$ for $f \in L^{1}\left(\mathbb{R}^{n}, d \mu\right)$ and $f \geq 0$ in Theorem 1.4, we have the following results.

Corollary 1.5 Let $0<\alpha<n, \beta \geq 0$ and $q=n /(n-\alpha)$. Then for any $f \in L^{1}\left(\mathbb{R}^{n}, d \mu\right)$ and $f \geq 0$,

(i) $\lim _{\lambda \rightarrow 0+} \lambda^{q} \mu\left(\left\{x \in \mathbb{R}^{n}: I_{\mu}^{\alpha} f(x)>\lambda\right\}\right)=\frac{\omega_{n-1}}{(n+\beta) \mu\left(B\left(e_{1}, 1\right)\right)}\|f\|_{L^{1}\left(\mathbb{R}^{n}, d \mu\right)}^{q}$;

(ii) $\lim _{\lambda \rightarrow 0+} \lambda^{q} \mu\left(\left\{x \in \mathbb{R}^{n}:\left|I_{\mu}^{\alpha} f(x)-\frac{\|f\|_{L^{1}\left(\mathbb{R}^{n}, d \mu\right)}}{\mu(B(x,|x|))^{1-\alpha / n}}\right|>\lambda\right\}\right)=0$.

The paper is organized as follows. In Section 2, after giving an auxiliary lemma, we prove Theorem 1.1. The proof of Theorem 1.4 will be given in Section 3.

Throughout this paper, the letter $C$, sometimes with additional parameters, will stand for positive constants, not necessarily the same one at each occurrence, but independent of the essential variables.

\section{Proof of Theorem 1.1}

This section is concerned with the proof of Theorem 1.1. At first, we present an auxiliary lemma, which will be used in the later arguments.

Lemma 2.1 Let $0 \leq \alpha<n, q=n /(n-\alpha)$, and let $e_{1}=(1,0, \ldots, 0)$ be a unit vector on $\mathbb{S}^{n-1}$. For a fixed $\lambda>0$, we have

$$
\mu\left(\left\{x \in \mathbb{R}^{n}: \frac{1}{\mu(B(x,|x|))^{1 / q}}>\lambda\right\}\right)=\frac{\omega_{n-1}}{\lambda^{q}(n+\beta) \mu\left(B\left(e_{1}, 1\right)\right)} .
$$

Proof It is easy to check that

$$
\begin{aligned}
\mu\left(\left\{x \in \mathbb{R}^{n}: \frac{1}{\mu(B(x,|x|))^{1 / q}}>\lambda\right\}\right) & =\mu\left(\left\{x \in \mathbb{R}^{n}:|x|^{n+\beta}<\frac{1}{\lambda q \mu\left(B\left(e_{1}, 1\right)\right)}\right\}\right) \\
& =\int_{\mathbb{S}^{n-1}} \int_{0}^{\left(\frac{1}{\lambda^{q} \mu\left(B\left(e_{1}, 1\right)\right)}\right)^{1 /(n+\beta)}} r^{n+\beta-1} d r d \sigma\left(x^{\prime}\right) \\
& =\frac{\omega_{n-1}}{\lambda^{q}(n+\beta) \mu\left(B\left(e_{1}, 1\right)\right)} .
\end{aligned}
$$

This completes the proof of Lemma 2.1.

Now we are in a position to prove Theorem 1.1.

Proof of Theorem 1.1 Without loss of generality, we can assume that $V\left(\mathbb{R}^{n}\right)=1$. Then for any $0<\varepsilon<1$, there exists $r_{\varepsilon}>0$ such that

$$
V\left(\left\{x \in \mathbb{R}^{n}:|x|<r_{\varepsilon}\right\}\right)>1-\varepsilon .
$$


Set $d V_{1}(x):=\chi_{B\left(0, r_{\varepsilon}\right)}(x) d V(x)$ and $d V_{2}(x):=\chi_{B\left(0, r_{\varepsilon}\right)^{c}}(x) d V(x)$. We get

$$
V_{1}\left(\mathbb{R}^{n}\right)>1-\varepsilon \quad \text { and } \quad V_{2}\left(\mathbb{R}^{n}\right)<\varepsilon
$$

For $\lambda>0$, we denote that

$$
\begin{aligned}
& E_{\lambda}=\left\{x \in \mathbb{R}^{n}: M_{\mu}^{\alpha} V(x)>\lambda\right\}, \\
& E_{\lambda}^{1}=\left\{x \in \mathbb{R}^{n}: M_{\mu}^{\alpha} V_{1}(x)>\lambda\right\}, \\
& E_{\lambda}^{2}=\left\{x \in \mathbb{R}^{n}: M_{\mu}^{\alpha} V_{2}(x)>\lambda\right\} .
\end{aligned}
$$

Note that $0<\sqrt{\varepsilon} \ll 1$, and then $E_{\lambda} \subseteq E_{(1-\sqrt{\varepsilon}) \lambda}^{1} \cup E_{\sqrt{\varepsilon} \lambda}^{2}$ and $E_{(1+\sqrt{\varepsilon}) \lambda}^{1} \subseteq E_{\lambda} \cup E_{\sqrt{\varepsilon} \lambda}^{2}$. We have

$$
\mu\left(E_{(1+\sqrt{\varepsilon}) \lambda}^{1}\right)-\mu\left(E_{\sqrt{\varepsilon} \lambda}^{2}\right) \leq \mu\left(E_{\lambda}\right) \leq \mu\left(E_{(1-\sqrt{\varepsilon}) \lambda}^{1}\right)+\mu\left(E_{\sqrt{\varepsilon} \lambda}^{2}\right) .
$$

Recalling that operator $M_{\mu}^{\alpha}$ is bounded from $L^{1}\left(\mathbb{R}^{n}, d \mu\right)$ to $L^{q, \infty}\left(\mathbb{R}^{n}, d \mu\right)$, we obtain that

$$
\mu\left(E_{\sqrt{\varepsilon} \lambda}^{2}\right) \leq\left(\frac{C V_{2}\left(\mathbb{R}^{n}\right)}{\sqrt{\varepsilon} \lambda}\right)^{q} \leq \frac{C \varepsilon^{q / 2}}{\lambda^{q}} .
$$

Consequently,

$$
\mu\left(E_{(1+\sqrt{\varepsilon}) \lambda}^{1}\right)-\frac{C \varepsilon^{q / 2}}{\lambda^{q}} \leq \mu\left(E_{\lambda}\right) \leq \mu\left(E_{(1-\sqrt{\varepsilon}) \lambda}^{1}\right)+\frac{C \varepsilon^{q / 2}}{\lambda^{q}} .
$$

Now we give the upper estimate of $\mu\left(E_{(1-\sqrt{\varepsilon}) \lambda}^{1}\right)$. Let $R_{\varepsilon}:=(1+1 / \varepsilon) r_{\varepsilon}$. Then we can write $E_{(1-\sqrt{\varepsilon}) \lambda}^{1}=E_{(1-\sqrt{\varepsilon}) \lambda}^{1,1} \cup E_{(1-\sqrt{\varepsilon}) \lambda}^{1,2}$, where

$$
\begin{aligned}
& E_{(1-\sqrt{\varepsilon}) \lambda}^{1,1}:=\left\{|x|>R_{\varepsilon}: M_{\mu}^{\alpha} V_{1}(x)>(1-\sqrt{\varepsilon}) \lambda\right\}, \\
& E_{(1-\sqrt{\varepsilon}) \lambda}^{1,2}:=\left\{|x| \leq R_{\varepsilon}: M_{\mu}^{\alpha} V_{1}(x)>(1-\sqrt{\varepsilon}) \lambda\right\} .
\end{aligned}
$$

For $|x|>R_{\varepsilon}$, it is easy to see that

$$
\begin{aligned}
\frac{1-\varepsilon}{\mu\left(B\left(x,|x|+r_{\varepsilon}\right)\right)^{1 / q}} & \leq M_{\mu}^{\alpha} V_{1}(x)=\sup _{r>0} \frac{V_{1}(B(x, r))}{\mu(B(x, r))^{1 / q}} \\
& \leq \frac{1}{\mu\left(B\left(x,|x|-r_{\varepsilon}\right)\right)^{1 / q}} .
\end{aligned}
$$

For $\mu\left(E_{(1-\sqrt{\varepsilon}) \lambda}^{1,1}\right)$, since $|x|-r_{\varepsilon}>|x| /(1+\varepsilon)$, it follows from Lemma 2.1 that

$$
\begin{aligned}
\mu\left(E_{(1-\sqrt{\varepsilon}) \lambda}^{1,1}\right) & \leq \mu\left(\left\{|x|>R_{\varepsilon}: \frac{1}{\mu\left(B\left(x,|x|-r_{\varepsilon}\right)\right)^{1 / q}}>(1-\sqrt{\varepsilon}) \lambda\right\}\right) \\
& \leq \mu\left(\left\{|x|>R_{\varepsilon}: \frac{1}{\mu(B(x,|x| /(1+\varepsilon)))^{1 / q}}>(1-\sqrt{\varepsilon}) \lambda\right\}\right) \\
& \leq \mu\left(\left\{x \in \mathbb{R}^{n}: \frac{(1+\varepsilon)^{n+\beta}}{\mu\left(B\left((\varepsilon+1) e_{1}, 1\right)\right)|x|^{n+\beta}}>(1-\sqrt{\varepsilon})^{q} \lambda^{q}\right\}\right)
\end{aligned}
$$




$$
\begin{aligned}
& \leq \mu\left(\left\{x \in \mathbb{R}^{n}:|x|^{n+\beta}<\frac{(\varepsilon+1)^{n+\beta}}{(1-\sqrt{\varepsilon})^{q} \lambda^{q} \mu\left(B\left(e_{1}, 1\right)\right)}\right\}\right) \\
& =\frac{\omega_{n-1}(\varepsilon+1)^{n+\beta}}{(1-\sqrt{\varepsilon})^{q} \lambda^{q}(n+\beta) \mu\left(B\left(e_{1}, 1\right)\right)} .
\end{aligned}
$$

Invoking (2.1), we deduce

$$
\begin{aligned}
\mu\left(E_{\lambda}\right) & \leq \mu\left(E_{(1-\sqrt{\varepsilon}) \lambda}^{1,1}\right)+\frac{C \varepsilon^{q / 2}}{\lambda^{q}}+\frac{\omega_{n-1} R_{\varepsilon}^{n+\beta}}{n+\beta} \\
& \leq \frac{\omega_{n-1}(\varepsilon+1)^{n+\beta}}{(1-\sqrt{\varepsilon})^{q} \lambda^{q}(n+\beta) \mu\left(B\left(e_{1}, 1\right)\right)}+\frac{C \varepsilon^{q / 2}}{\lambda^{q}}+\frac{\omega_{n-1} R_{\varepsilon}^{n+\beta}}{n+\beta} .
\end{aligned}
$$

Then by letting $\lambda \rightarrow 0+$ and the arbitrariness of $\varepsilon$, we get

$$
\varlimsup_{\lambda \rightarrow 0+} \lambda^{q} \mu\left(E_{\lambda}\right) \leq \frac{\omega_{n-1}}{(n+\beta) \mu\left(B\left(e_{1}, 1\right)\right)} .
$$

Next we turn to the lower estimate of $\mu\left(E_{\lambda}\right)$. Note that

$$
\mu\left(E_{(1+\sqrt{\varepsilon}) \lambda}^{1}\right) \geq \mu\left(\left\{|x|>R_{\varepsilon}: M_{\mu}^{\alpha} V_{1}(x)>(1+\sqrt{\varepsilon}) \lambda\right\}\right) .
$$

In view of (2.2) and $|x|+r_{\varepsilon} \leq(2 \varepsilon+1)|x| /(\varepsilon+1)$, we have

$$
\begin{aligned}
\mu\left(E_{(1+\sqrt{\varepsilon}) \lambda}^{1}\right) & \geq \mu\left(\left\{|x|>R_{\varepsilon}: \frac{1-\varepsilon}{\mu\left(B\left(x,|x|+r_{\varepsilon}\right)\right)^{1 / q}}>(1+\sqrt{\varepsilon}) \lambda\right\}\right) \\
& \geq \mu\left(\left\{|x|>R_{\varepsilon}: \frac{1-\varepsilon}{\mu(B(x,(2 \varepsilon+1)|x| /(\varepsilon+1)))^{1 / q}}>(1+\sqrt{\varepsilon}) \lambda\right\}\right) \\
& \geq \mu\left(\left\{|x|>R_{\varepsilon}: \frac{\mu\left(B\left(\frac{\varepsilon+1}{2 \varepsilon+1} e_{1}, 1\right)(2 \varepsilon+1)^{n+\beta}|x|^{n+\beta}\right.}{(1+\varepsilon)^{n+\beta}}<\frac{(1-\varepsilon)^{q}}{(1+\sqrt{\varepsilon})^{q} \lambda^{q}}\right\}\right) \\
& \geq \mu\left(\left\{x \in \mathbb{R}^{n}:|x|^{n+\beta}<\frac{(1-\varepsilon)^{q}(1+\varepsilon)^{n+\beta}(2 \varepsilon+1)^{-n-\beta}}{\lambda^{q}(1+\sqrt{\varepsilon})^{q} \mu\left(B\left(e_{1}, 1\right)\right)}\right\}\right)-\frac{\omega_{n-1} R_{\varepsilon}^{n+\beta}}{n+\beta} \\
& =\frac{\omega_{n-1}(1-\varepsilon)^{q}(1+\varepsilon)^{n+\beta}}{\lambda^{q}(n+\beta)(1+\sqrt{\varepsilon})^{q}(2 \varepsilon+1)^{n+\beta} \mu\left(B\left(e_{1}, 1\right)\right)}-\frac{\omega_{n-1} R_{\varepsilon}^{n+\beta}}{n+\beta} .
\end{aligned}
$$

Hence,

$$
\begin{aligned}
\mu\left(E_{\lambda}\right) & \geq \mu\left(E_{(1+\sqrt{\varepsilon}) \lambda}^{1}\right)-\frac{C \varepsilon^{q / 2}}{\lambda^{q}} \\
& \geq \frac{\omega_{n-1}(1-\varepsilon)^{q}(1+\varepsilon)^{n+\beta}}{\lambda q(n+\beta)(1+\sqrt{\varepsilon})(2 \varepsilon+1)^{n+\beta} \mu\left(B\left(e_{1}, 1\right)\right)}-\frac{\omega_{n-1} R_{\varepsilon}^{n+\beta}}{n+\beta}-\frac{C \varepsilon^{q / 2}}{\lambda^{q}} .
\end{aligned}
$$

By letting $\lambda \rightarrow 0+$ and the arbitrariness of $\varepsilon$ again, we get

$$
\varliminf_{\lambda \rightarrow 0+} \lambda^{q} \mu\left(E_{\lambda}\right) \geq \frac{\omega_{n-1}}{(n+\beta) \mu\left(B\left(e_{1}, 1\right)\right)} .
$$

This combined with (2.3) implies that

$$
\lim _{\lambda \rightarrow 0+} \lambda^{q} \mu\left(E_{\lambda}\right)=\frac{\omega_{n-1}}{(n+\beta) \mu\left(B\left(e_{1}, 1\right)\right)}
$$

and completes the proof of conclusion (i). 
We now prove the conclusion (ii). For $\lambda>0$, set

$$
\begin{aligned}
& G_{\lambda}:=\left\{x \in \mathbb{R}^{n}:\left|M_{\mu}^{\alpha} V(x)-\frac{1}{\mu(B(x,|x|))^{1 / q}}\right|>\lambda\right\}, \\
& G_{\lambda}^{1}:=\left\{|x|>R_{\varepsilon}:\left|M_{\mu}^{\alpha} V_{1}(x)-\frac{1}{\mu(B(x,|x|))^{1 / q}}\right|>\lambda\right\} .
\end{aligned}
$$

It is not difficult to verify that

$$
\begin{aligned}
\mu\left(G_{\lambda}\right) & \leq \mu\left(G_{\left(1-\varepsilon^{1 /(2 q)}\right) \lambda}^{1}\right)+\mu\left(\left\{x \in \mathbb{R}^{n}: M_{\mu}^{\alpha} V_{2}(x)>\varepsilon^{1 /(2 q)} \lambda\right\}\right)+\frac{\omega_{n-1} R_{\varepsilon}^{n+\beta}}{n+\beta} \\
& \leq \mu\left(G_{\left(1-\varepsilon^{1 /(2 q)}\right) \lambda}^{1}\right)+\frac{C \varepsilon^{q-1 / 2}}{\lambda^{q}}+\frac{\omega_{n-1} R_{\varepsilon}^{n+\beta}}{n+\beta} .
\end{aligned}
$$

Therefore, it remains to estimate $\mu\left(G_{\left(1-\varepsilon^{1 /(2 q)}\right) \lambda}^{1}\right)$. Set

$$
\begin{aligned}
& K_{1}(x):=\frac{1}{\mu\left(B\left(x,|x|-r_{\varepsilon}\right)\right)^{1 / q}}-\frac{1-\varepsilon}{\mu\left(B\left(x,|x|+r_{\varepsilon}\right)\right)^{1 / q}}, \\
& K_{2}(x):=\frac{1}{\mu\left(B\left(x,|x|-r_{\varepsilon}\right)\right)^{1 / q}}-\frac{1}{\mu(B(x,|x|))^{1 / q}} .
\end{aligned}
$$

Using (2.2), we have

$$
\begin{aligned}
\mu\left(G_{\left(1-\varepsilon^{1 /(2 q)}\right) \lambda}^{1}\right) & \\
\leq & \mu\left(\left\{|x|>R_{\varepsilon}:\left|M_{\mu}^{\alpha} V_{1}(x)-\frac{1}{\mu\left(B\left(x,|x|-r_{\varepsilon}\right)\right)^{1 / q}}\right|>\left(1-2 \varepsilon^{1 /(2 q)}\right) \lambda\right\}\right) \\
& +\mu\left(\left\{|x|>R_{\varepsilon}:\left|\frac{1}{\mu\left(B\left(x,|x|-r_{\varepsilon}\right)\right)^{1 / q}}-\frac{1}{\mu(B(x,|x|))^{1 / q}}\right|>\varepsilon^{1 /(2 q)} \lambda\right\}\right) \\
= & \mu\left(\left\{|x|>R_{\varepsilon}:\left|K_{1}(x)\right|>\left(1-2 \varepsilon^{1 /(2 q)}\right) \lambda\right\}\right) \\
& +\mu\left(\left\{|x|>R_{\varepsilon}:\left|K_{2}(x)\right|>\varepsilon^{1 /(2 q)} \lambda\right\}\right) .
\end{aligned}
$$

It follows from $|x|>R_{\varepsilon}=(\varepsilon+1) r_{\varepsilon} / \varepsilon$ by definition that

$$
|x|-r_{\varepsilon}>|x| /(\varepsilon+1) \text { and }|x|+r_{\varepsilon}<|x|(2 \varepsilon+1) /(\varepsilon+1) .
$$

Hence,

$$
\begin{aligned}
\left|K_{1}(x)\right| & \leq \frac{\left|\mu\left(B\left(x,|x|+r_{\varepsilon}\right)\right)^{1 / q}-\mu\left(B\left(x,|x|-r_{\varepsilon}\right)\right)^{1 / q}\right|+\varepsilon \mu\left(B\left(x,|x|-r_{\varepsilon}\right)\right)^{1 / q}}{\mu\left(B\left(x,|x|+r_{\varepsilon}\right)\right)^{1 / q} \mu\left(B\left(x,|x|-r_{\varepsilon}\right)\right)^{1 / q}} \\
& \leq \frac{\left|\mu\left(B\left(x,|x|+r_{\varepsilon}\right)\right)^{1 / q}-\mu\left(B\left(x,|x|-r_{\varepsilon}\right)\right)^{1 / q}\right|}{\mu\left(B\left(x,|x|+r_{\varepsilon}\right)\right)^{1 / q} \mu\left(B\left(x,|x|-r_{\varepsilon}\right)\right)^{1 / q}}+\frac{\varepsilon}{\mu\left(B\left(x,|x|+r_{\varepsilon}\right)\right)^{1 / q}} \\
& =: K_{1}^{1}(x)+\frac{\varepsilon}{\mu\left(B\left(x,|x|+r_{\varepsilon}\right)\right)^{1 / q}} .
\end{aligned}
$$


By the fact that $\left|a^{\gamma}-b^{\gamma}\right| \leq|a-b|^{\gamma}$ for any $a, b \geq 0,0<\gamma<1$, we get

$$
\begin{aligned}
\left|K_{1}^{1}(x)\right| & \leq \frac{\left|\int_{B(x,(2 \varepsilon+1)|x| /(\varepsilon+1))}\right| y||^{\beta} d y-\left.\int_{B(x,|x| /(\varepsilon+1))}|y|^{\beta} d y\right|^{1 / q}}{\mu(B(x,|x|))^{1 / q} \mu(B(x,|x| /(\varepsilon+1)))^{1 / q}} \\
& \leq \frac{\left.\left|(2 \varepsilon+1)^{n+\beta} \int_{B\left((\varepsilon+1) e_{1} /(2 \varepsilon+1), 1\right)}\right| y\right|^{\beta} d y-\left.\int_{B\left((\varepsilon+1) e_{1}, 1\right)}|y|^{\beta} d y\right|^{1 / q}}{\left(\left.\int_{B\left(e_{1}, 1\right)}|y|^{\beta} d y \int_{B\left((\varepsilon+1) e_{1}, 1\right)}|y|\right|^{\beta} d y|x|^{n+\beta}\right)^{1 / q}} \\
& \leq \frac{\left((2 \varepsilon+1)^{n+\beta}-1\right)^{1 / q}}{|x|^{(n+\beta) / q} \mu\left(B\left(e_{1}, 1\right)\right)^{1 / q}} .
\end{aligned}
$$

Then

$$
\left|K_{1}(x)\right| \leq \frac{\left((2 \varepsilon+1)^{n+\beta}-1\right)^{1 / q}}{|x|^{(n+\beta) / q} \mu\left(B\left(e_{1}, 1\right)\right)^{1 / q}}+\frac{\varepsilon}{\mu(B(x,|x|))^{1 / q}} .
$$

Similarly, we have

$$
\begin{aligned}
\left|K_{2}(x)\right| & \leq\left|\frac{1}{\mu\left(B\left(x,|x|-r_{\varepsilon}\right)\right)}-\frac{1}{\mu(B(x,|x|))}\right|^{1 / q} \\
& \leq\left|\frac{\int_{B(x,|x|)}|y|^{\beta} d y-\int_{B(x,|x| /(\varepsilon+1))}|y|^{\beta} d y}{\int_{B(x,|x|)}|y|^{\beta} d y \int_{B(x,|x| /(\varepsilon+1))}|y|^{\beta} d y}\right|^{1 / q} \leq\left|\frac{(\varepsilon+1)^{n+\beta}-1}{|x|^{n+\beta} \mu\left(B\left(e_{1}, 1\right)\right)}\right|^{1 / q} .
\end{aligned}
$$

Therefore,

$$
\begin{aligned}
\mu\left(G_{\left(1-\varepsilon^{1 /(2 q)}\right) \lambda}^{1} \leq\right. & \mu\left(\left\{x \in \mathbb{R}^{n}: \frac{\left((2 \varepsilon+1)^{n+\beta}-1\right)^{1 / q}}{|x|^{(n+\beta) / q} \mu\left(B\left(e_{1}, 1\right)\right)^{1 / q}}>\left(1-3 \varepsilon^{1 /(2 q)}\right) \lambda\right\}\right) \\
& +\mu\left(\left\{x \in \mathbb{R}^{n}: \frac{\varepsilon}{\mu(B(x,|x|))^{1 / q}}>\varepsilon^{1 /(2 q)} \lambda\right\}\right) \\
& +\mu\left(\left\{x \in \mathbb{R}^{n}:\left|\frac{(\varepsilon+1)^{n+\beta}-1}{|x|^{n+\beta} \mu\left(B\left(e_{1}, 1\right)\right)}\right|^{1 / q}>\varepsilon^{1 /(2 q)} \lambda\right\}\right) \\
\leq & \frac{\omega_{n-1}\left((2 \varepsilon+1)^{n+\beta}-1\right)}{\lambda q\left(1-3 \varepsilon^{1 /(2 q)}\right)^{q} \mu\left(B\left(e_{1}, 1\right)\right)}+\frac{\omega_{n-1} \varepsilon^{q-1 / 2}}{\lambda q \mu\left(B\left(e_{1}, 1\right)\right)} \\
& +\frac{\omega_{n-1}\left((\varepsilon+1)^{n+\beta}-1\right)}{\sqrt{\varepsilon} \lambda q \mu\left(B\left(e_{1}, 1\right)\right)} .
\end{aligned}
$$

Combining with the above estimates, we obtain

$$
\begin{aligned}
\mu\left(G_{\lambda}\right) \leq & \mu\left(G_{\left(1-\varepsilon^{1 /(2 q)}\right) \lambda}^{1}\right)+\frac{C \varepsilon^{q-1 / 2}}{\lambda q}+\frac{\omega_{n-1} R_{\varepsilon}^{n+\beta}}{n+\beta} \\
\leq & \frac{\omega_{n-1}\left((2 \varepsilon+1)^{n+\beta}-1\right)}{\lambda^{q}\left(1-3 \varepsilon^{1 /(2 q)}\right)^{q} \mu\left(B\left(e_{1}, 1\right)\right)}+\frac{\omega_{n-1} \varepsilon^{q-1 / 2}}{\lambda q \mu\left(B\left(e_{1}, 1\right)\right)} \\
& +\frac{\omega_{n-1}\left((\varepsilon+1)^{n+\beta}-1\right)}{\sqrt{\varepsilon} \lambda^{q} \mu\left(B\left(e_{1}, 1\right)\right)}+\frac{C \varepsilon^{q-1 / 2}}{\lambda^{q}}+\frac{\omega_{n-1} R_{\varepsilon}^{n+\beta}}{n+\beta} .
\end{aligned}
$$

Now letting $\lambda \rightarrow 0+$ and noting that $\varepsilon$ is arbitrary, we obtain

$$
\lim _{\lambda \rightarrow 0+} \lambda^{q} \mu\left(G_{\lambda}\right)=0
$$

and complete the proof of conclusion (ii). 
Next, we verify conclusion (iii), using similar arguments with some appropriate modifications. For $|x|>R_{\varepsilon}, x^{\prime}=x /|x|$, it is easy to check that

$$
\begin{aligned}
\frac{1-\varepsilon}{\mu\left(B\left(\left(x-r_{\varepsilon} x^{\prime}\right) / 2,\left(|x|+r_{\varepsilon}\right) / 2\right)\right)^{1 / q}} & \leq \widetilde{M}_{\mu}^{\alpha} V_{1}(x)=\sup _{B_{x} \ni x} \frac{V_{1}\left(B_{x}\right)}{\mu\left(B_{x}\right)^{1 / q}} \\
& \leq \frac{1}{\mu\left(B\left(\left(x+r_{\varepsilon} x^{\prime}\right) / 2,\left(|x|-r_{\varepsilon}\right) / 2\right)\right)^{1 / q}} .
\end{aligned}
$$

From this, by similar arguments as in the proof of the conclusion (i), we can get similar upper and lower estimates with $\widetilde{M}_{\mu}^{\alpha}$ substituted for $M_{\mu}^{\alpha}$. Moreover, note that $\mu(B(x,|x|))^{1 / q}=2^{(n+\beta) / q} \mu(B(x / 2,|x| / 2))^{1 / q}$, and we can get conclusion (iii).

Furthermore, the proof of conclusion (iv) is quite similar to that of conclusion (ii). We omit the details here.

Finally, conclusion (v) follows directly from conclusions (ii) and (iv). Theorem 1.1 is proved.

Remark 2.2 We remark that Theorem 1.1(ii) is stronger than conclusion (i).

Indeed, for $\lambda>0$, let $G_{\lambda}$ be as in (2.4) in the proof of Theorem 1.1. Then for $0<$ $\varepsilon \ll \eta<1$, by Lemma 2.1, we have

$$
\begin{aligned}
\mu\left(\left\{x \in \mathbb{R}^{n}: M_{\mu}^{\alpha} V(x)>\lambda\right\}\right) & \leq \mu\left(G_{\eta \lambda}\right)+\mu\left(\left\{x \in \mathbb{R}^{n}: \frac{1}{\mu(B(x,|x|))^{1 / q}}>(1-\eta) \lambda\right\}\right) \\
& =\mu\left(G_{\eta \lambda}\right)+\frac{\omega_{n-1}}{(1-\eta)^{q} \lambda^{q}(n+\beta) \mu\left(B\left(e_{1}, 1\right)\right)} .
\end{aligned}
$$

By (2.5) and letting $\lambda \rightarrow 0+, \varepsilon \rightarrow 0$ and $\eta \rightarrow 0$, we know that

$$
\varlimsup_{\lambda \rightarrow 0+} \lambda^{q} \mu\left(\left\{x \in \mathbb{R}^{n}: M_{\mu}^{\alpha} V(x)>\lambda\right\}\right) \leq \frac{\omega_{n-1}}{(n+\beta) \mu\left(B\left(e_{1}, 1\right)\right)} .
$$

On the other hand,

$$
\begin{aligned}
\mu\left(\left\{x \in \mathbb{R}^{n}: M_{\mu}^{\alpha} V(x)>\lambda\right\}\right) & \geq \mu\left(\left\{x \in \mathbb{R}^{n}: \frac{1}{\mu(B(x,|x|))^{1 / q}}>(1+\eta) \lambda\right\}\right)-\mu\left(G_{\eta \lambda}\right) \\
& =\frac{\omega_{n-1}}{(1+\eta)^{q} \lambda^{q}(n+\beta) \mu\left(B\left(e_{1}, 1\right)\right)}-\mu\left(G_{\eta \lambda}\right) .
\end{aligned}
$$

Now invoking (2.5) and letting $\lambda \rightarrow 0+, \varepsilon \rightarrow 0$, and $\eta \rightarrow 0$, we get

$$
\varliminf_{\lambda \rightarrow 0+} \lambda^{q} \mu\left(\left\{x \in \mathbb{R}^{n}: M_{\mu}^{\alpha} V(x)>\lambda\right\}\right) \geq \frac{\omega_{n-1}}{(n+\beta) \mu\left(B\left(e_{1}, 1\right)\right)} .
$$

Combining with (2.6) and (2.7), we obtain conclusion (i).

\section{Proof of Theorem 1.4}

This section is devoted to proving Theorem 1.4. Without loss of generality, we can assume that $V\left(\mathbb{R}^{n}\right)=1$. Then for any $0<\varepsilon \ll 1$, there exists $r_{\varepsilon}>0$ such that

$$
V\left(\left\{x \in \mathbb{R}^{n}:|x|<r_{\varepsilon}\right\}\right)>1-\varepsilon .
$$


Denote $d V_{1}(x)=\chi_{\left\{x \in \mathbb{R}^{n}:|x|<r_{\varepsilon}\right\}}(x) d V(x)$ and $d V_{2}(x)=\chi_{\left\{x \in \mathbb{R}^{n}:|x| \geq r_{\varepsilon}\right\}}(x) d V(x)$. We know that $V_{1}\left(\mathbb{R}^{n}\right)>1-\varepsilon$ and $V_{2}\left(\mathbb{R}^{n}\right)<\varepsilon$. Set

$$
\begin{aligned}
& F_{\lambda}=\left\{x \in \mathbb{R}^{n}:\left|I_{\mu}^{\alpha} V(x)\right|>\lambda\right\}, \\
& F_{\lambda}^{1}=\left\{x \in \mathbb{R}^{n}:\left|I_{\mu}^{\alpha} V_{1}(x)\right|>\lambda\right\}, \\
& F_{\lambda}^{2}=\left\{x \in \mathbb{R}^{n}:\left|I_{\mu}^{\alpha} V_{2}(x)\right|>\lambda\right\} .
\end{aligned}
$$

Note that $0<\varepsilon<\varepsilon^{1 /(2 q)} \ll 1$, and we have

$$
F_{\lambda} \subseteq F_{\left(1-\varepsilon^{1 /(2 q)}\right) \lambda}^{1} \cup F_{\varepsilon^{1 /(2 q)} \lambda}^{2} \text {, and } \quad F_{\left(1+\varepsilon^{1 / 2 q}\right) \lambda}^{1} \subseteq F_{\lambda} \cup F_{\varepsilon^{1 /(2 q)} \lambda}^{2} .
$$

Then

$$
\mu\left(F_{\left(1+\varepsilon^{1 /(2 q)}\right) \lambda}^{1}\right)-\mu\left(F_{\varepsilon^{1 /(2 q) \lambda}}^{2}\right) \leq \mu\left(F_{\lambda}\right) \leq \mu\left(F_{\left(1-\varepsilon^{1 /(2 q)}\right) \lambda}^{1}\right)+\mu\left(F_{\varepsilon^{1 /(2 q)} \lambda}^{2}\right) .
$$

By the $\left(L^{1}\left(\mathbb{R}^{n}, d \mu\right), L^{q, \infty}\left(\mathbb{R}^{n}, d \mu\right)\right)$-boundedness of $I_{\mu}^{\alpha}$, we get

$$
\mu\left(F_{\varepsilon^{1 /(2 q) \lambda}}^{2}\right) \leq \frac{C}{\sqrt{\varepsilon} \lambda^{q}} V_{2}\left(\mathbb{R}^{n}\right)^{q} \leq \frac{C \varepsilon^{q}}{\sqrt{\varepsilon} \lambda^{q}} \leq \frac{C \varepsilon^{q-1 / 2}}{\lambda^{q}},
$$

which leads to

$$
\mu\left(F_{\left(1+\varepsilon^{1 /(2 q)}\right) \lambda}^{1}\right)-\frac{C \varepsilon^{q-1 / 2}}{\lambda^{q}} \leq \mu\left(F_{\lambda}\right) \leq \mu\left(F_{\left(1-\varepsilon^{1 /(2 q)}\right) \lambda}^{1}\right)+\frac{C \varepsilon^{q-1 / 2}}{\lambda^{q}} .
$$

Next, we will estimate $\mu\left(F_{\left(1-\varepsilon^{1 /(2 q)}\right) \lambda}^{1}\right)$ and $\mu\left(F_{\left(1+\varepsilon^{1 /(2 q)}\right) \lambda}^{1}\right)$, respectively. Employing the notation $R_{\varepsilon}=(1+1 / \mathcal{E}) r_{\varepsilon}$ as in the proof of Theorem 1.1, we can write $F_{\lambda}^{1}=F_{\lambda}^{1,1} \cup F_{\lambda}^{1,2}$, where

$$
F_{\lambda}^{1,1}:=\left\{|x|>R_{\varepsilon}:\left|I_{\mu}^{\alpha} V_{1}(x)\right|>\lambda\right\} \quad \text { and } \quad F_{\lambda}^{1,2}:=\left\{|x| \leq R_{\varepsilon}:\left|I_{\mu}^{\alpha} V_{1}(x)\right|>\lambda\right\} .
$$

We first estimate $\mu\left(F_{\left(1-\varepsilon^{1 /(2 q)}\right) \lambda}^{1,1}\right)$. Set

$$
L(x, y):=\frac{1}{\mu(B(x,|x-y|))^{1 / q}}-\frac{1}{\mu(B(x,|x|))^{1 / q}} .
$$

Then

$$
\begin{aligned}
\mu\left(F_{\left(1-\varepsilon^{1 /(2 q)}\right) \lambda}^{1,1}\right) \leq & \mu\left(\left\{|x|>R_{\varepsilon}: \int_{\mathbb{R}^{n}}|L(x, y)| d V_{1}(y)>\varepsilon^{1 /(2 q)} \lambda\right\}\right) \\
& +\mu\left(\left\{|x|>R_{\varepsilon}: \frac{1}{\mu(B(x,|x|))^{1 / q}}>\left(1-2 \varepsilon^{1 /(2 q)}\right) \lambda\right\}\right) \\
=: & J_{1}+J_{2} .
\end{aligned}
$$

By Lemma 2.1, we conclude that

$$
\begin{aligned}
J_{2} & \leq \mu\left(\left\{x \in \mathbb{R}^{n}: \frac{1}{\mu(B(x,|x|))^{1 / q}}>\left(1-2 \varepsilon^{1 /(2 q)}\right) \lambda\right\}\right) \\
& =\frac{\omega_{n-1}}{(n+\beta)\left(\lambda\left(1-2 \varepsilon^{1 /(2 q)}\right)\right)^{q} \mu\left(B\left(e_{1}, 1\right)\right)} .
\end{aligned}
$$

Now we estimate $J_{1}$. 
(i) When $|x| \geq|x-y|$, since $|x|>R_{\varepsilon},|y|<r_{\varepsilon}$, we have

$$
|x| /(\varepsilon+1)<|x-y|<(2 \varepsilon+1)|x| /(\varepsilon+1) .
$$

Recall that $\left|a^{\gamma}-b^{\gamma}\right| \leq|a-b|^{\gamma}$ for any $a, b \geq 0,0<\gamma<1$. Then the mean value theorem tells us that

$$
\begin{aligned}
|L(x, y)| & \leq \frac{\left.\left|\int_{B(x,|x|)}\right| z\right|^{\beta} d z-\left.\int_{B(x,|x-y|)}|z|^{\beta} d z\right|^{1 / q}}{\left(\int_{B(x,|x|)}|z|^{\beta} d z \int_{B(x,|x-y|)}|z|^{\beta} d z\right)^{1 / q}} \\
& \leq\left(\frac{|x|^{n+\beta}-|x-y|^{n+\beta}}{\mu\left(B\left(e_{1}, 1\right)\right)|x|^{2 n+2 \beta}(\varepsilon+1)^{-(n+\beta)}}\right)^{1 / q} \leq\left(\frac{C|y|}{|x|^{n+\beta+1} \mu\left(B\left(e_{1}, 1\right)\right)}\right)^{1 / q} .
\end{aligned}
$$

(ii) When $|x|<|x-y|$, we can similarly deduce that

$$
|L(x, y)| \leq\left(\frac{|x-y|^{n+\beta}-|x|^{n+\beta}}{\mu\left(B\left(e_{1}, 1\right)\right)|x-y|^{2 n+2 \beta}(\varepsilon+1)^{-(n+\beta)}}\right)^{1 / q} \leq\left(\frac{C|y|}{|x|^{n+\beta+1} \mu\left(B\left(e_{1}, 1\right)\right)}\right)^{1 / q} .
$$

Hence,

$$
\begin{aligned}
J_{1} & \leq \frac{1}{\sqrt{\varepsilon} \lambda^{q}} \int_{|x|>R_{\varepsilon}}\left(\int_{\mathbb{R}^{n}}|L(x, y)| d V_{1}(y)\right)^{q} d \mu(x) \\
& \leq \frac{C r_{\varepsilon}}{\sqrt{\varepsilon} \lambda^{q}} \int_{|x|>R_{\varepsilon}} \frac{1}{|x|^{n+\beta+1} \mu\left(B\left(e_{1}, 1\right)\right)} d \mu(x) \\
& \leq \frac{C r_{\varepsilon}}{\sqrt{\varepsilon} \lambda^{q} \mu\left(B\left(e_{1}, 1\right)\right)} \int_{|x|>R_{\varepsilon}} \frac{1}{|x|^{n+1}} d x \leq \frac{C r_{\varepsilon}}{\sqrt{\varepsilon} \lambda^{q} \mu\left(B\left(e_{1}, 1\right)\right) R_{\varepsilon}} .
\end{aligned}
$$

Combining the above estimates for $J_{1}$ and $J_{2}$, we obtain

$$
\mu\left(F_{\left(1-\varepsilon^{1 /(2 q)}\right) \lambda}^{1,1}\right) \leq \frac{C r_{\varepsilon}}{\sqrt{\varepsilon} \lambda q \mu\left(B\left(e_{1}, 1\right)\right) R_{\varepsilon}}+\frac{\omega_{n-1}}{(n+\beta)\left(\lambda\left(1-2 \varepsilon^{1 /(2 q)}\right)\right)^{q} \mu\left(B\left(e_{1}, 1\right)\right)},
$$

which together with(3.2) implies that

$$
\begin{aligned}
\mu\left(F_{\lambda}\right) \leq & \mu\left(F_{\left(1-\varepsilon^{1 /(2 q)}\right) \lambda}^{1,1}\right)+\frac{C \varepsilon^{q-1 / 2}}{\lambda^{q}}+\frac{\omega_{n-1} R_{\varepsilon}^{n+\beta}}{n+\beta} \\
\leq & \frac{C r_{\varepsilon}}{\sqrt{\varepsilon} \lambda q \mu\left(B\left(e_{1}, 1\right)\right) R_{\varepsilon}}+\frac{\omega_{n-1}}{(n+\beta)\left(\lambda\left(1-2 \varepsilon^{1 /(2 q)}\right)\right)^{q} \mu\left(B\left(e_{1}, 1\right)\right)} \\
& +\frac{C \varepsilon^{q-1 / 2}}{\lambda^{q}}+\frac{\omega_{n-1} R_{\varepsilon}^{n+\beta}}{n+\beta} .
\end{aligned}
$$

Note that $r_{\varepsilon} / R_{\varepsilon}=\varepsilon /(1+\varepsilon)$. By letting $\lambda \rightarrow 0+$ and the arbitrariness of $\varepsilon$, we get

$$
\varlimsup_{\lambda \rightarrow 0+} \lambda^{q} \mu\left(F_{\lambda}\right) \leq \frac{\omega_{n-1}}{(n+\beta) \mu\left(B\left(e_{1}, 1\right)\right)} .
$$

On the other hand, we have

$$
\begin{aligned}
\mu\left(F_{\left(1+\varepsilon^{1 /(2 q)} \lambda\right.}^{1}\right) & \geq \mu\left(\left\{|x|>R_{\varepsilon}:\left|I_{\mu}^{\alpha} V_{1}(x)\right|>\left(1+\varepsilon^{1 /(2 q)}\right) \lambda\right\}\right) \\
& =\mu\left(\left\{|x|>R_{\varepsilon}: \int_{\mathbb{R}^{n}} \frac{1}{\mu(B(x,|x-y|))^{1 / q}} d V_{1}(y)>\left(1+\varepsilon^{1 /(2 q)}\right) \lambda\right\}\right) .
\end{aligned}
$$


Since

$$
\begin{aligned}
\left|\int_{\mathbb{R}^{n}} \frac{1}{\mu(B(x,|x|))^{1 / q}} d V_{1}(y)\right| \leq & \int_{\mathbb{R}^{n}}\left|\frac{1}{\mu(B(x,|x-y|))^{1 / q}}-\frac{1}{\mu(B(x,|x|))^{1 / q}}\right| d V_{1}(y) \\
& +\left|\int_{\mathbb{R}^{n}} \frac{1}{\mu(B(x,|x-y|))^{1 / q}} d V_{1}(y)\right| \\
& =\int_{\mathbb{R}^{n}}|L(x, y)| d V_{1}(y)+\int_{\mathbb{R}^{n}} \frac{d V_{1}(y)}{\mu(B(x,|x-y|))^{1 / q}}
\end{aligned}
$$

we have

$$
\begin{aligned}
\mu\left(\left\{|x|>R_{\varepsilon}:\left|\int_{\mathbb{R}^{n}} \frac{1}{\mu(B(x,|x|))^{1 / q}} d V_{1}(y)\right|>\left(1+2 \varepsilon^{1 /(2 q)}\right) \lambda\right\}\right) \\
\leq \mu\left(\left\{|x|>R_{\varepsilon}:\left|\int_{\mathbb{R}^{n}} \frac{1}{\mu(B(x,|x-y|))^{1 / q}} d V_{1}(y)\right|>\left(1+\varepsilon^{1 /(2 q)}\right) \lambda\right\}\right) \\
\quad+\mu\left(\left\{|x|>R_{\varepsilon}: \int_{\mathbb{R}^{n}}|L(x, y)| d V_{1}(y)>\varepsilon^{1 /(2 q)} \lambda\right\}\right) .
\end{aligned}
$$

This together with Lemma 2.1 and (3.3) leads to

$$
\begin{aligned}
\mu\left(F_{\left(1+\varepsilon^{1 /(2 q)}\right) \lambda}^{1} \geq\right. & \mu\left(\left\{|x|>R_{\varepsilon}:\left|\int_{\mathbb{R}^{n}} \frac{1}{\mu(B(x,|x|))^{1 / q}} d V_{1}(y)\right|>\left(1+2 \varepsilon^{1 /(2 q)}\right) \lambda\right\}\right) \\
& -\mu\left(\left\{|x|>R_{\varepsilon}: \int_{\mathbb{R}^{n}}|L(x, y)| d V_{1}(y)>\varepsilon^{1 /(2 q)} \lambda\right\}\right) \\
= & \mu\left(\left\{x \in \mathbb{R}^{n}:\left|\int_{\mathbb{R}^{n}} \frac{1}{\mu(B(x,|x|))^{1 / q}} d V_{1}(y)\right|>\left(1+2 \varepsilon^{1 /(2 q)}\right) \lambda\right\}\right) \\
& -\mu\left(\left\{|x| \leq R_{\varepsilon}:\left|\int_{\mathbb{R}^{n}} \frac{1}{\mu(B(x,|x|))^{1 / q}} d V_{1}(y)\right|>\left(1+2 \varepsilon^{1 /(2 q)}\right) \lambda\right\}\right) \\
& -\mu\left(\left\{|x|>R_{\varepsilon}: \int_{\mathbb{R}^{n}}|L(x, y)| d V_{1}(y)>\varepsilon^{1 /(2 q)} \lambda\right\}\right) \\
\geq & \frac{\omega_{n-1}(1-\varepsilon)^{q}}{(n+\beta)\left(\lambda\left(2 \varepsilon^{1 / 2 q}+1\right)\right)^{q} \mu\left(B\left(e_{1}, 1\right)\right)}-\frac{\omega_{n-1} R_{\varepsilon}^{n+\beta}}{n+\beta} \\
& -\frac{C r_{\varepsilon}}{\sqrt{\varepsilon} \lambda q \mu\left(B\left(e_{1}, 1\right)\right) R_{\varepsilon}} .
\end{aligned}
$$

By letting $\lambda \rightarrow 0+$ and the arbitrariness of $\varepsilon$ again, we get

$$
\varliminf_{\lambda \rightarrow 0+} \lambda^{q} \mu\left(F_{\lambda}\right) \geq \frac{\omega_{n-1}}{(n+\beta) \mu\left(B\left(e_{1}, 1\right)\right)} .
$$

Combining (3.4) with (3.5) yields that

$$
\lim _{\lambda \rightarrow 0} \lambda^{q} \mu\left(\left\{x \in \mathbb{R}^{n}:\left|I_{\mu}^{\alpha} V(x)\right|>\lambda\right\}\right)=\frac{\omega_{n-1}}{(n+\beta) \mu\left(B\left(e_{1}, 1\right)\right)},
$$

which completes the proof of conclusion (i). 
Next, we turn to proving conclusion (ii). Note that

$$
\begin{aligned}
& \left|I_{\mu}^{\alpha} V(x)-\frac{V\left(\mathbb{R}^{n}\right)}{\mu(B(x,|x|))^{1 / q}}\right| \\
& \quad \leq\left|I_{\mu}^{\alpha} V(x)-\frac{V_{1}\left(\mathbb{R}^{n}\right)}{\mu(B(x,|x|))^{1 / q}}\right|+\frac{V_{2}\left(\mathbb{R}^{n}\right)}{\mu(B(x,|x|))^{1 / q}} \\
& \quad \leq\left|I_{\mu}^{\alpha} V_{1}(x)-\frac{V_{1}\left(\mathbb{R}^{n}\right)}{\mu(B(x,|x|))^{1 / q}}\right|+\left|I_{\mu}^{\alpha} V_{2}(x)\right|+\frac{\varepsilon}{\mu(B(x,|x|))^{1 / q}} \\
& \quad \leq \int_{\mathbb{R}^{n}}|L(x, y)| d V_{1}(y)+\left|I_{\mu}^{\alpha} V_{2}(x)\right|+\frac{\varepsilon}{\mu(B(x,|x|))^{1 / q}},
\end{aligned}
$$

where $L(x, y)$ is as before. Recalling (3.1), Lemma 2.1, and (3.3), we have

$$
\begin{aligned}
\mu(\{x \in & \left.\left.\mathbb{R}^{n}:\left|I_{\mu}^{\alpha} V(x)-\frac{V\left(\mathbb{R}^{n}\right)}{\mu(B(x,|x|))^{1 / q}}\right|>\lambda\right\}\right) \\
\leq & \mu\left(\left\{|x|>R_{\varepsilon}:\left|I_{\mu}^{\alpha} V(x)-\frac{V\left(\mathbb{R}^{n}\right)}{\mu(B(x,|x|))^{1 / q}}\right|>\lambda\right\}\right)+\frac{\omega_{n-1} R_{\varepsilon}^{n+\beta}}{n+\beta} \\
\leq & \mu\left(\left\{|x|>R_{\varepsilon}: \int_{\mathbb{R}^{n}}|L(x, y)| d V_{1}(y)>\varepsilon^{1 /(2 q)} \lambda\right\}\right) \\
& +\mu\left(\left\{|x|>R_{\varepsilon}:\left|I_{\mu}^{\alpha} V_{2}(x)\right|>\varepsilon^{1 /(2 q)} \lambda\right\}\right) \\
& +\mu\left(\left\{|x|>R_{\varepsilon}: \frac{\varepsilon}{\mu(B(x,|x|))^{1 / q}}>\left(1-2 \varepsilon^{1 /(2 q)}\right) \lambda\right\}\right)+\frac{\omega_{n-1} R_{\varepsilon}^{n+\beta}}{n+\beta} \\
\leq & \frac{C r_{\varepsilon}}{\sqrt{\varepsilon} \lambda q \mu\left(B\left(e_{1}, 1\right)\right) R_{\varepsilon}}+\frac{C \varepsilon^{q-1 / 2}}{\lambda q} \\
& +\frac{\omega_{n-1} \varepsilon^{q}}{(n+\beta)\left(\lambda\left(1-2 \varepsilon^{1 / 2 q}\right)\right)^{q} \mu\left(B\left(e_{1}, 1\right)\right)}+\frac{\omega_{n-1} R_{\varepsilon}^{n+\beta}}{n+\beta} .
\end{aligned}
$$

Recall that $r_{\varepsilon} / R_{\varepsilon}=\varepsilon /(1+\varepsilon)$; by letting $\lambda \rightarrow 0+$ and noting that $\varepsilon$ is arbitrary, we obtain

$$
\lim _{\lambda \rightarrow 0+} \lambda^{q} \mu\left(\left\{x \in \mathbb{R}^{n}:\left|I_{\mu}^{\alpha} V(x)-\frac{V\left(\mathbb{R}^{n}\right)}{\mu(B(x,|x|))^{1-\alpha / n}}\right|>\lambda\right\}\right)=0 .
$$

This completes the proof of Theorem 1.4.

\section{References}

[1] R. Coifman and G. Weiss, Analyse harmonique non-commutative sur certains espaces homogènes. Lecture Note in Mathematics, 242, Springer-Verlag, Berlin-New York, 1971.

[2] Y. Ding and X. Lai, $L^{1}$-Dini conditions and limiting behavior of weak type estimates for singular integrals. Rev. Mat. Iberoam. 33(2017), no. 4, 1267-1284. http://dx.doi.org/10.4171/RMI/971

[3] — Weak type $(1,1)$ behavior for the maximal operator with $L^{1}$-Dini kernel. Potential Anal. 47(2017), no. 2, 169-187. http://dx.doi.org/10.1007/s11118-017-9612-3

[4] A. Gatto, C. Gutiérrez, and R. Wheeden, On weighted fractional integrals. In: Conference on harmonic analysis in honor of Antoni Zygmund, Vol. I, II (Chicago, III, 1981), Wadsworth Math. Ser., Wadsworth, Belmont, CA, 1983, pp. 124-137.

[5] J. Heinonen, Lectures on analysis on metric spaces. Universitext, Springer-Verlag, New York, 2001. http://dx.doi.org/10.1007/978-1-4613-0131-8

[6] J. Hu and X. Huang, A note on the limiting weak-type behavior for maximal operators. Proc. Amer. Math. Soc. 136(2008), 1599-1607. http://dx.doi.org/10.1090/S0002-9939-08-09313-1 
X. Hou and $\mathrm{H}$. Wu

[7] P. Janakiraman, Limiting weak-type behavior for singular integral and maximal operators. Trans. Amer. Math. Soc. 358(2006), no. 5, 1937-1952. http://dx.doi.org/10.1090/S0002-9947-05-04097-3

[8] W. J. Pan, Fractional integrals on spaces of homogeneous type. Approx. Theory Appl. 8(1992), 1-15.

School of Mathematical Sciences, Xiamen University, Xiamen 361005, China

e-mail: houxianming37@163.com huoxwu@xmu.edu.cn 